\title{
The Car Following Model with Relative Speed in Front on the Three-Lane Road
}

\author{
Zichu Gao $\mathbb{C}^{1},{ }^{1}$ Ning Zhang, ${ }^{1}$ Livia Mannini, ${ }^{2}$ and Ernesto Cipriani ${ }^{2}$ \\ ${ }^{1}$ School of Economics and Management, Beihang University, Beijing 100083, China \\ ${ }^{2}$ Department of Engineering, Roma Tre University, 00185 Rome, Italy \\ Correspondence should be addressed to Zichu Gao; zhuzhusenlin@163.com
}

Received 3 November 2017; Accepted 19 February 2018; Published 10 April 2018

Academic Editor: Antonio Iannizzotto

Copyright (c) 2018 Zichu Gao et al. This is an open access article distributed under the Creative Commons Attribution License, which permits unrestricted use, distribution, and reproduction in any medium, provided the original work is properly cited.

An improved car following model on one road with three lanes is presented in this paper, which considers the relative velocity in front on the main lane and the left and the right adjacent lanes. The stability criterion and neutral stability curve are obtained by linear stability theory. The nonlinear stability analysis is investigated further to get the solution of the modified Korteweg-de Vries $(\mathrm{mKdV})$ equation and get the three areas of stability, metastability, and unstability. The new LRVD model (left and right lane velocity difference model) with bigger stable area can stabilize middle lane traffic flow better, which is proved by the linear theory, nonlinear theory, and the simulation. The LRVD model shows if drivers on the middle lane pay more attention to more cars in front on the two side lanes on the three-lane road, the middle lane traffic flow is certain to be more stable in real life. On the complex three-lane road, if intelligent traffic management system based on the huge traffic data for drivers is applied in real life, it is very helpful to ensure traffic safety, which is also the trend of transportation development in future.

\section{Introduction}

With the development of the economy, transportation has made great progress. The road congestion problem especially has attracted more and more attention. Therefore, the relationship of vehicles in traffic flow becomes an importation issue. Car following theory is a main method to study cars behavior in the microscopic traffic flow. In 1995, Bando et al. [1] proposed an optimal velocity model, OV car following model. A lot of important models on one single road appeared [2-16]. In 2000, Jiang et al. [5] proposed the full speed difference car following model (FVD), which was based on the positive and negative speed difference. Li et al. [15] proposed an improved model reconsidering the influence of safety distance and velocity difference on the acceleration of following car. Then Yu et al. [17] proposed the LVD model to explain cars behavior on the two-lane road, which indeed enhances the stability of model to describe the real phenomenon. However limited research has been done which considers the three-lane road in the real traffic situation. Chen et al. [18] proposed a three-lane traffic flow model to investigate the effect of car accidents on the traffic flow. The extended lane-changing rules are presented in this model. The cases where the car accidents occupy the exterior or interior lane, the medium lane, and two lanes are studied by numerical simulations. The results show that the car accident has a different effect on the traffic flow when it occupies different lanes. The car accidents have a more serious effect on the whole road when they occupy two lanes. The larger the density is, the greater the influence on the traffic flow becomes. Until now scholars have not considered how the cars behavior is affected by the relative speed in front on the three lanes of one road. In fact, with the development of society, more and more roads with high traffic capacity have been developed to adapt to the rapid social life. So this research on the three-lane road is very necessary. But no existing models can explain the complicated phenomenon that cars behavior on the middle lane with cars driving in front of the three lanes of one road in real life, but which is more common. If the cars ahead on the left or right lane slow down, the following driver on the middle lane may think the signal light in front has turned red, or there are some traffic accidents or traffic jam in front. This will cause the cars on the middle lane to also slow down. If the cars ahead on the 
left or right lane speed up, the drivers on the middle lane may believe a smaller number of cars run in front, which causes the driver to increase the speed too, or the drivers on the middle lane would like to decrease their speed, since they want to avoid cars on the adjacent lanes having to change lanes which could cause an accident when some emergency appears in front on the adjacent lane. On the three-lane road especially, cars' acceleration may appear on one adjacent lane and deceleration phenomenon could happen on the other adjacent lane at the same time, which must be more complicated. So it is meaningful for us to study the complex phenomenon above on three lanes by considering the relative speed ahead on the three lanes on one road to explain these complex phenomena in this paper.

In this paper, the new LRVD model is proposed, which studies the cars behavior on the middle lane of one road with three lanes. In Section 2, the LRVD model is elaborated in detail. In Section 3, the linear analysis theory of LRVD model, including the neutral curve, is presented. In Section 4, by studying the nonlinear theory, we get the coexisting curve of the model, which divides the traffic flow into stable, metastable, and unstable regions. In Section 5, simulation results also prove LRVD model indeed enhances the stability of the traffic flow. The last section concludes some remarks of LRVD model.

\section{Model}

At the very beginning, Chowdhury et al. [4] proposed car following model on one single road just as follows:

$$
\frac{d x_{n}^{2}}{d t^{2}}=f_{\text {sti }}\left(v_{n}, \Delta x_{n}, \Delta v_{n}\right)
$$

where the function $f_{\text {sti }}(\cdot)$ represents the drivers' response to the stimulus, which is related to the speed of $n$th car $v_{n}$, the relative speed between the cars in front of the $n$th car and the $n$th car, and the distance between them.

Newell [6] proposed a simple single road car following model.

$$
\frac{d x_{n}(x+\tau)}{d t}=V\left(\Delta x_{n}(t)\right)
$$

where $V\left(\Delta x_{n}(t)\right)$ is the optimal velocity function, which just contains the distance between the $n$th car and the car in front. $\tau$ is the delay time; the driver of the $n$th car can respond to the stimulus of the $(n+1)$ th car in front after the time lag $\tau$.

$\mathrm{Yu}$ [8] considered that the optimal velocity may be determined not only by the successive space but also by the response to the stimulus of the relative velocity. In this case, $d x_{n}(t+\tau) / d t=V\left(\Delta x_{n}(t), \Delta v_{n}(t)\right)$, which further describes the car following process as follows, namely, RV model:

$$
\frac{d^{2} x_{n}(t)}{d t^{2}}=\alpha\left[V\left(\Delta x_{n}(t)\right)-v_{n}(t)\right]+k_{1} \Delta v_{n}(t) .
$$

Yu et al. [17] proposed the LVD model based on the RV model, which considers the impact from the relative velocity on the main and adjacent lanes. By expanding (2) into $d x_{n}(t+\tau) / d t=V\left(\Delta x_{n}(t), \Delta v_{n}(t), \Delta v_{n}{ }^{\text {LVD }}(t)\right)$, and assuming $V\left(\Delta x_{n}(t), \Delta v_{n}(t), \Delta v_{n}{ }^{\mathrm{LVD}}(t)\right)=V\left(\Delta x_{n}(t)\right)+\lambda_{1} \Delta v_{n}(t)+$ $\lambda_{2} \tau \Delta v_{n}{ }^{\mathrm{LVD}}(t)$, the LVD model is obtained as follows:

$$
\begin{aligned}
\frac{d^{2} x_{n}(t)}{d t^{2}}= & \alpha\left[V\left(\Delta x_{n}(t)\right)-v_{n}(t)\right]+k_{1} \Delta v_{n}(t) \\
& +\lambda_{2} \Delta v_{n}{ }^{\mathrm{LVD}}(t) .
\end{aligned}
$$

The scholars have improved the car following model in many aspects before; however no paper was taken with regard to the cars behavior on the middle lane of the three-lane road. In fact, one road with three same lanes, as Figure 1 has shown, is more common than one single road or two-lane road in real life. Considering the complex phenomena above, the LRVD model is proposed in this paper. Equation (2) can be expanded into such differential equation:

$$
\begin{aligned}
& \frac{d x_{n}(t+\tau)}{d t} \\
& =V\left(\Delta x_{n}(t), \Delta v_{n}(t), \Delta v_{n}^{\mathrm{LVD}}(t), \Delta v_{n}^{\mathrm{RVD}}(t)\right),
\end{aligned}
$$

where $\Delta v_{n}^{\mathrm{LVD}}(t)=\operatorname{mean}\left(v_{m}^{L}, v_{m+1}^{L}, \ldots, v_{m+s-1}^{L}\right)-v_{n}$, and $\Delta v_{n}^{\mathrm{RVD}}(t)=\operatorname{mean}\left(v_{k}^{R}, v_{k+1}^{R}, \ldots, v_{k+s-1}^{R}\right)-v_{n} . v_{n}$ is the speed of the $n$th car on the main lane. $s$ is the car number on the left lane and the right lane. The mean $\left(v_{m}^{L}, v_{m+1}^{L}, \ldots, v_{m+s-1}^{L}\right)$ is the average speed of all the cars on the left lane. The mean $\left(v_{k}^{R}, v_{k+1}^{R}, \ldots, v_{k+s-1}^{R}\right)$ is the average speed of all the cars on the right lane.

Here we suppose the linear combination of $V\left(\Delta x_{n}(t)\right.$, $\left.\Delta v_{n}(t), \Delta v_{n}^{\mathrm{LVD}}(t), \Delta v_{n}^{\mathrm{RVD}}(t)\right)$ :

$$
\begin{aligned}
V( & \left.\Delta x_{n}(t), \Delta v_{n}(t), \Delta v_{n}^{\mathrm{LVD}}(t), \Delta v_{n}^{\mathrm{RVD}}(t)\right) \\
= & V\left(\Delta x_{n}(t)\right)+\lambda_{1} \Delta v_{n}(t)+\lambda_{2} \tau \Delta v_{n}{ }^{\mathrm{LVD}}(t) \\
& +\lambda_{3} \tau \Delta v_{n}^{\mathrm{RVD}}(t) .
\end{aligned}
$$

Then after applying Talor expanding to (5), we get the following model:

$$
\begin{aligned}
\frac{d^{2} x_{n}(t)}{d t^{2}}= & \alpha\left[V\left(\Delta x_{n}(t)\right)-v_{n}(t)\right]+k_{1} \Delta v_{n}(t) \\
& +\lambda_{2} \Delta v_{n}{ }^{\mathrm{LVD}}(t)+\lambda_{3} \Delta v_{n}^{\mathrm{RVD}}(t),
\end{aligned}
$$

where $k_{1}=\lambda_{1} / \tau$ and $\lambda_{1}$ is the response coefficient of the relative speed between the $n$th car and the $(n+1)$ th car in front. $\lambda_{2}$ is the response coefficient of the relative speed between cars on the main and left adjacent lane, which are just in front of the $n$th car. $\lambda_{3}$ is the response coefficient of the relative speed between cars on the main and right adjacent lanes, which are just in front of the $n$th car.

$V\left(\Delta x_{n}(t)\right)$ is the optimal velocity of $n$th car at time $t$, which is defined as follows:

$$
V\left(\Delta x_{n}\right)=\frac{v_{\max }}{2}\left(\tanh \left(\Delta x_{n}-h_{c}\right)+\tanh \left(h_{c}\right)\right),
$$

where $h_{c}$ is the safe distance of successive cars and $v_{\max }$ is the maximum velocity. Note that when we ignore the relative 


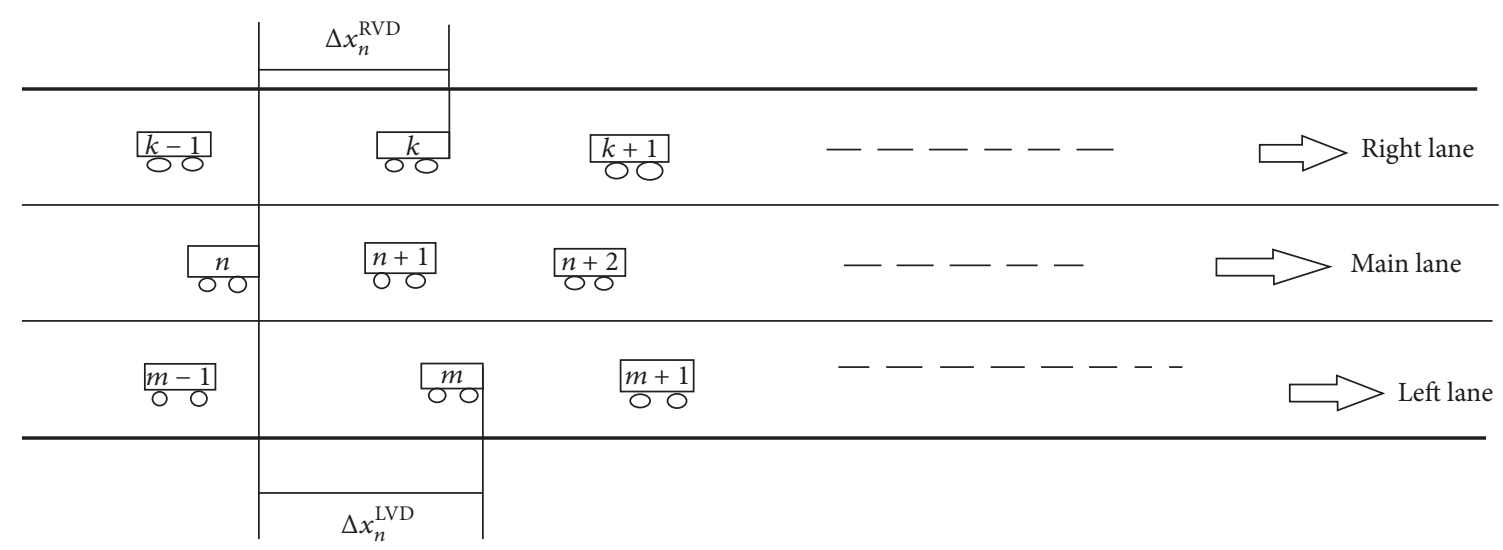

FIGURE 1: Three-lane road traffic situation.

speed on the right adjacent lane, namely, $\lambda_{3}=0$, the LRVD model proposed can be degenerated to the LVD model. Then if we also ignore the relative speed on the left adjacent lane and the right adjacent lane, $\lambda_{2}=\lambda_{3}=0$, the LRVD model proposed can be degenerated to the RV model. Further, if we continue to ignore the relative speed on the main lane, namely, $\lambda_{1}=\lambda_{2}=\lambda_{3}=0$, the new LRVD model can be degenerated into the original OV model. So the theory continuity of the OV, RV, LVD and LRVD models indicates the LRVD model proposed is reasonable and it can indeed explain some complicated phenomenon on three-lane road that scholars have not studied before. In a word, LRVD model is the basement of the three-lane road traffic phenomenon.

The dynamic equation (7) can be discretized by the asymmetric forward difference, so we get

$$
\begin{aligned}
x_{n}(t+2 \tau)= & x_{n}(t+\tau)+\tau V\left(\Delta x_{n}(t)\right) \\
& +\lambda_{1}\left(\Delta x_{n}(t+\tau)-\Delta x_{n}(t)\right) \\
& +\lambda_{2} \tau\left(\frac{1}{s} \sum_{j=1}^{s} \Delta x_{m+j-1}^{L}(t)-\Delta x_{n}(t)\right) \\
& +\lambda_{3} \tau\left(\frac{1}{s} \sum_{j=1}^{s} \Delta x_{k+j-1}^{L}(t)-\Delta x_{n}(t)\right) .
\end{aligned}
$$

Here, $\lambda_{1}, \lambda_{2}$, and $\lambda_{3}$ are defined as follows:

$$
\begin{aligned}
& \lambda_{1}= \begin{cases}\pi, & \Delta x_{n}<s_{c}, \\
0, & \Delta x_{n} \geq s_{c},\end{cases} \\
& \lambda_{2}= \begin{cases}\eta, & \Delta x_{m}^{L}<s_{c}, \\
0, & \Delta x_{m}^{L} \geq s_{c},\end{cases} \\
& \lambda_{3}= \begin{cases}\phi, & \Delta x_{k}^{R}<s_{c}, \\
0, & \Delta x_{k}^{R} \geq s_{c},\end{cases}
\end{aligned}
$$

where $\pi, \eta, \phi$ are the given response coefficients and $s_{c}$ is a critical successive space. If the distance between the two successive cars on the main lane is more than $\Delta x_{n}$, the speed difference of these two cars would not affect the following car's behavior at all. If the distance between the car on the main lane and the car in front of it on the left adjacent lane is beyond the critical successive space, these two cars' relative speed can be ignored. If the distance between the car on the main lane and the car in front of it on the right adjacent lane is more than the critical successive space $s_{c}$, these two cars' relative speed can be ignored together.

Eq. (9) can be rewritten as

$$
\begin{aligned}
& \Delta x_{n}(t+2 \tau)=\Delta x_{n}(t+\tau)+\tau V\left(\Delta x_{n+1}(t)\right) \\
& -\tau V\left(\Delta x_{n}(t)\right)+\lambda_{1}\left(\Delta x_{n+1}(t+\tau)-\Delta x_{n+1}(t)\right. \\
& \left.-\Delta x_{n}(t+\tau)+\Delta x_{n}(t)\right) \\
& +\lambda_{2} \tau\left[\left(\frac{1}{s} \sum_{j=1}^{s} \Delta x_{m+j}^{L}(t)-\Delta x_{m+j-1}^{L}(t)\right)\right. \\
& \left.-\left(\Delta x_{n+1}(t)-\Delta x_{n}(t)\right)\right] \\
& +\lambda_{3} \tau\left[\left(\frac{1}{s} \sum_{j=1}^{s} \Delta x_{k+j}^{R}(t)-\Delta x_{k+j-1}^{R}(t)\right)\right. \\
& \left.-\left(\Delta x_{n+1}(t)-\Delta x_{n}(t)\right)\right] .
\end{aligned}
$$

\section{Linear Stability Analysis}

The hypotheses of LRVD model are (a) cars cannot change their lane, (b) the three lanes on one road are the same, (c) neither overtaking nor ramp exists in this model, and (d) all the cars on the right and left adjacent lanes can be affected by the cars in front on the middle lane and also on their own respective lanes. 
Here we study the uniform traffic flow stability on each lane, which is given as the solution of the uniformly steady state of (9), which is shown as

$$
\begin{aligned}
& x_{n}^{(0)}(t)=h n+V(h) t, \quad h=\frac{L}{N_{1}}, \\
& x_{m}^{L(0)}(t)=h_{l} m+V_{l}\left(h_{l}\right) t, \quad h_{l}=\frac{L}{N_{2}}, \\
& x_{k}^{R(0)}(t)=h_{r} k+V_{r}\left(h_{r}\right) t, \quad h_{r}=\frac{L}{N_{3}},
\end{aligned}
$$

where $N_{1}, N_{2}$ and $N_{3}$ represent the number of cars on the main lane, the left adjacent lane, and right adjacent lane; $h$, $h_{l}$, and $h_{r}$ are the successive space on the main lane, the left adjacent lane, and the right adjacent lane; $L$ is the length of the road; $V(h), V_{l}\left(h_{l}\right)$, and $V_{r}\left(h_{r}\right)$ indicate the optimal speed of the main lane, the left adjacent lane, and the right adjacent lane; $x_{n}^{(0)}(t), x_{m}^{L(0)}(t)$, and $x_{k}^{R(0)}(t)$ are the position of the $n$th car on the main lane, the $m$ th car on the left adjacent lane, and the $k$ th car on the right adjacent lane at $t=0$ in the stable state.

Considering the small perturbation into the steady state solutions in (12), $x_{n}(t)=x_{n}^{(0)}(t)+y_{n}(t), x_{m}^{L}(t)=x_{m}^{L(0)}(t)+$ $y_{m}^{L}(t)$, and the linear stability equation can be obtained:

$$
\begin{aligned}
& \Delta y_{n}(t+2 \tau)=\Delta y_{n}(t+\tau)+\tau V^{\prime}\left(\Delta y_{n+1}(t)-\Delta y_{n}(t)\right) \\
& +\lambda_{1}\left(\Delta y_{n+1}(t+\tau)-\Delta y_{n+1}(t)-\Delta y_{n}(t+\tau)\right. \\
& \left.+\Delta y_{n}(t)\right) \\
& +\lambda_{2} \tau\left[\left(\frac{1}{s} \sum_{j=1}^{s} \Delta y_{m+j}^{L}(t)-\Delta y_{m+j-1}^{L}(t)\right)\right. \\
& \left.-\left(\Delta y_{n+1}(t)-\Delta y_{n}(t)\right)\right] \\
& +\lambda_{3} \tau\left[\left(\frac{1}{s} \sum_{j=1}^{s} \Delta y_{k+j}^{R}(t)-\Delta y_{k+j-1}^{R}(t)\right)\right. \\
& \left.-\left(\Delta y_{n+1}(t)-\Delta y_{n}(t)\right)\right]
\end{aligned}
$$

where $V^{\prime}(h)=d V(\Delta x) / d \Delta x$. Expanding $\Delta y$ into the (13) with $\Delta y_{n}(t)=A \exp \{i k n+z t\}$,

$$
\begin{aligned}
& \Delta y_{m}^{L}(t) \cong \gamma A \exp \{i k n+z t\}, \\
& \Delta y_{k}^{R}(t) \cong \gamma A \exp \{i k n+z t\},
\end{aligned}
$$

where $\gamma$ represents the average strength coefficient which describes the perturbations between the main lane and the adjacent lanes. Then we get (13) as follows:

$$
\begin{aligned}
e^{2 z \tau}= & e^{z \tau}+V^{\prime}\left(e^{i k}-1\right) \tau+\lambda_{1}\left(e^{i k+z \tau}-e^{i k}-e^{z \tau}+1\right) \\
& +\lambda_{2} \tau\left[\frac{r}{s} \sum_{j=1}^{s}\left(e^{i k j}-e^{i k(j-1)}\right)-\left(e^{i k}-1\right)\right] \\
& +\lambda_{3} \tau\left[\frac{r}{s} \sum_{j=1}^{s}\left(e^{i k j}-e^{i k(j-1)}\right)-\left(e^{i k}-1\right)\right] .
\end{aligned}
$$

Inserting $z=z_{1}(i k)+z_{2}(i k)^{2}+\cdots$ into (15), we obtain

$$
\begin{aligned}
& z_{1}=V^{\prime}+\left(\lambda_{2}+\lambda_{3}\right)(\gamma-1), \\
& z_{2}=-\frac{3}{2} z_{1}^{2} \tau+\frac{V^{\prime}}{2}+\lambda_{1} z_{1}+\left(\lambda_{2}+\lambda_{3}\right) \frac{\gamma s-1}{2} .
\end{aligned}
$$

The flow is unstable if $z_{2}<0$, and stable if $z_{2}>0$. So the neutral stability condition is given by $z_{2}=0$ :

$$
\tau=\frac{V^{\prime}+2 \lambda_{1} z_{1}+\left(\lambda_{2}+\lambda_{3}\right)(\gamma s-1)}{3 z_{1}^{2}} .
$$

Considering the inverse of delay time $\tau$ which is the critical sensitivity $\alpha_{c}$, the neutral stability curve is shown as follows:

$$
=\frac{3\left[V^{\prime}+\left(\lambda_{2}+\lambda_{3}\right)(\gamma-1)\right]^{2}}{V^{\prime}+2 \lambda_{1}\left[V^{\prime}+\left(\lambda_{2}+\lambda_{3}\right)(\gamma-1)\right]+\left(\lambda_{2}+\lambda_{3}\right)(\gamma s-1)} .
$$$$
\alpha_{c}
$$

The unstable and stable areas are

$\alpha_{c}$

$$
<\frac{3\left[V^{\prime}+\left(\lambda_{2}+\lambda_{3}\right)(\gamma-1)\right]^{2}}{V^{\prime}+2 \lambda_{1}\left[V^{\prime}+\left(\lambda_{2}+\lambda_{3}\right)(\gamma-1)\right]+\left(\lambda_{2}+\lambda_{3}\right)(\gamma s-1)},
$$

$\alpha_{c}$

$$
>\frac{3\left[V^{\prime}+\left(\lambda_{2}+\lambda_{3}\right)(\gamma-1)\right]^{2}}{V^{\prime}+2 \lambda_{1}\left[V^{\prime}+\left(\lambda_{2}+\lambda_{3}\right)(\gamma-1)\right]+\left(\lambda_{2}+\lambda_{3}\right)(\gamma s-1)} .
$$

In Figure 2, suppose that the OV model with $\lambda_{1}=\lambda_{2}=$ $\lambda_{3}=0$, RV model with $\lambda_{1}=0.2, \lambda_{2}=\lambda_{3}=0$, the LVD model with $\lambda_{1}=\lambda_{2}=0.2, \lambda_{3}=0, \gamma=0.4$, the LRVD model with $\lambda_{1}=\lambda_{2}=\lambda_{3}=0.2, \gamma=0.4, s=1$, and $v_{\max }=3 \mathrm{~m} / \mathrm{s}$. Through Figure 2, we know the stable area of LRVD model is the biggest compared to others, which means considering the cars' relative speed on the third adjacent lane indeed improves the traffic flow stability. So it is necessary to propose the new LRVD model in this paper, which also can describe the real traffic phenomenon on the three-lane road in real life.

As Figure 3 has shown, if drivers on the middle lane pay more attention to the cars behavior on the two adjacent lanes, the stable area is much bigger. The more attention is paid, the more stable the area is. So if the drivers on the middle lane pay more attention to the cars' speed on the two side lanes, the traffic flow on the middle lane will be more stable. 


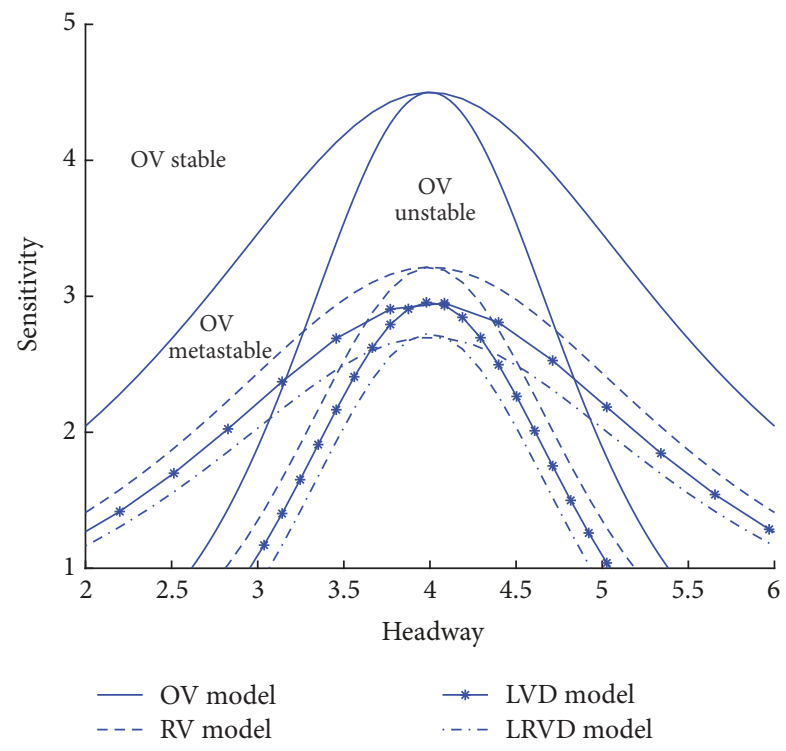

FIGURE 2: Neutral stability curves and coexisting curves of OV, RV, LVD, and LRVD models.

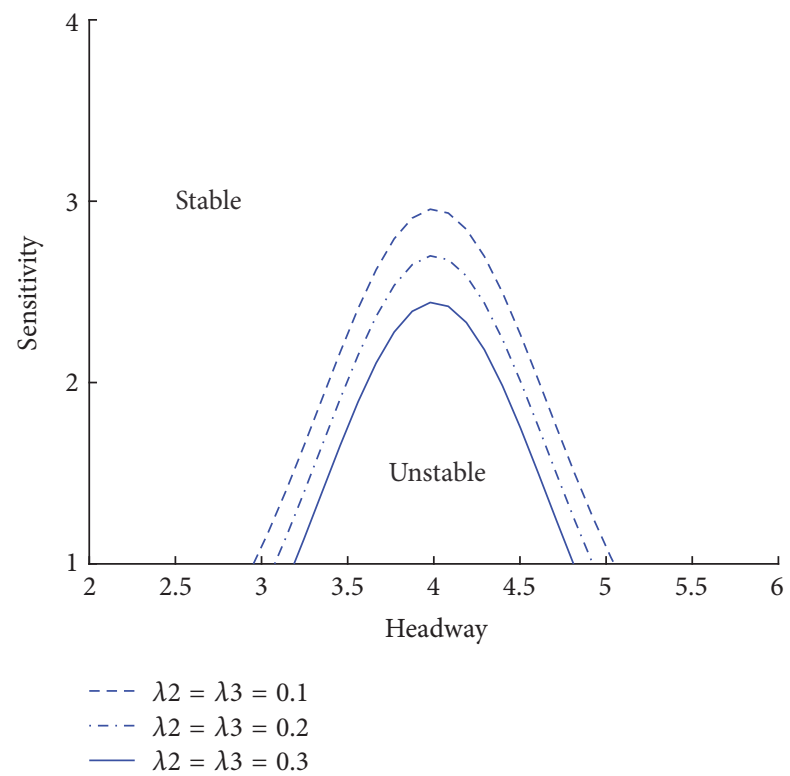

FIgURE 3: Different stable curves with different $\lambda_{2}$ and $\lambda_{3}$ in LVRD model.

As Figure 4 has shown, if the drivers on the middle lane pay the same attention to the two other lanes, the more cars on the two adjacent lanes are considered by the drivers on the middle lane and the more stable the traffic flow on the middle lane will be.

Through Figures 3 and 4, we realize the cars' driving truth on the three-lane road. Firstly, if the drivers on the middle lane pay more attention to the other lanes, rather than only see the cars on middle lane or one adjacent lane just like the existing model only on the single road or two-lane road, the traffic flow on the middle lane must be more stable. Secondly, in the same condition of the middle lane, if more cars in front on the two adjacent lanes are noticed by the driver on the

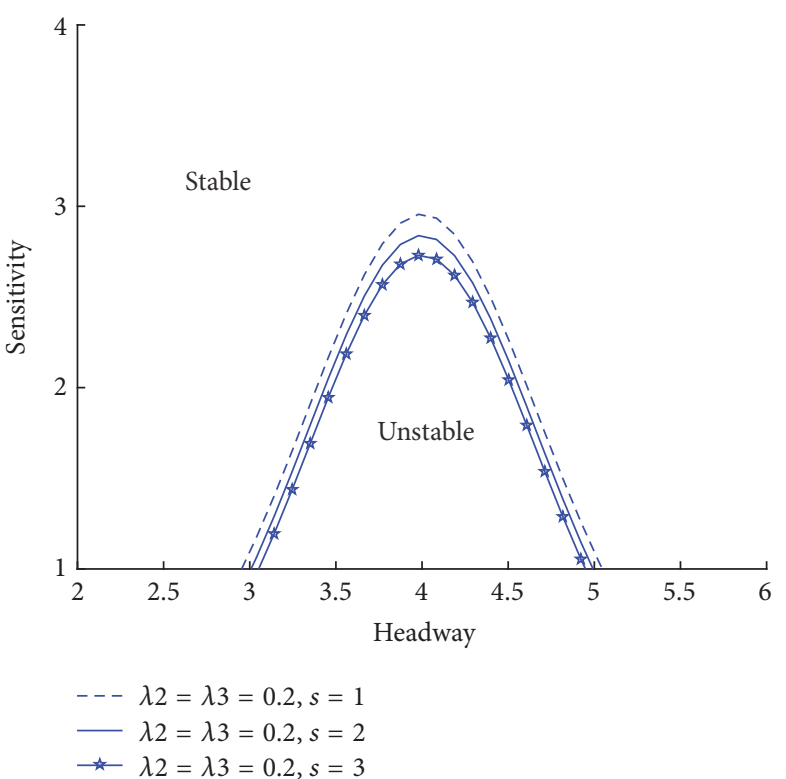

FIGURE 4: Different stable curves with different car numbers on the two adjacent lanes in LVRD model.

middle lane, the middle lane traffic flow is certain to be more stable.

\section{Nonlinear Stability Analysis}

To prove that the relative speed on the three lanes on one road indeed affects the traffic flow on the middle lane effectively, we conduct a nonlinear stability of the LRVD model. Here the nonlinear method is used to study the slowly varying behavior of long waves near the critical point $\left(h_{c}, a_{c}\right)$, and the slow scales for space variable $n$ and time variable $t$. The slow variables $X$ and $T$ are defined as follows:

$$
\begin{aligned}
& X=\varepsilon(n+b t), \\
& T=\varepsilon^{3} t,
\end{aligned}
$$

$$
0<\varepsilon \ll 1,
$$

where $b$ is a constant to be decided later. Considering a small disturbance $\varepsilon R(X, T)$, the distances between the two successive cars can be rewritten as follows:

$$
\Delta x_{n}(t)=h_{c}+\varepsilon R(X, T) .
$$

Then expanding (11) to the $\varepsilon$ fifth order by substituting (20) and (21), we can get the nonlinear partial differential equation as follows:

$$
\begin{gathered}
\varepsilon^{2}\left[b-V^{\prime}-\left(\lambda_{2}+\lambda_{3}\right)(\gamma-1)\right] \partial_{X} R+\varepsilon^{3}\left(\frac{3 b^{2} \tau}{2}-\frac{V^{\prime}}{2}\right. \\
\left.-\lambda_{1} b-\left(\lambda_{2}+\lambda_{3}\right) \frac{\gamma s-1}{2}\right) \partial_{X}^{2} R+\varepsilon^{4}\left[\partial_{T} R\right. \\
+\left(\frac{7}{6} b^{3} \tau^{2}-\frac{V^{\prime}}{6}-\frac{\lambda_{1} b(1+b \tau)}{2}\right.
\end{gathered}
$$




$$
\begin{aligned}
& \left.\left.-\left(\lambda_{2}+\lambda_{3}\right) \frac{r s^{2}-1}{6}\right) \partial_{X}^{3} R-\frac{V^{\prime \prime \prime}}{6} \partial_{X} R^{3}\right] \\
& +\varepsilon^{5}\left[\left(\frac{5}{8} b^{4} \tau^{3}-\frac{V^{\prime}}{24}-\lambda_{1} \frac{b\left(2 b^{2} \tau^{2}+3 b \tau+2\right)}{12}\right.\right. \\
& \left.\left.-\left(\lambda_{2}+\lambda_{3}\right) \frac{s^{3} \gamma-1}{24}\right) \partial_{X}^{4} R\right] \varepsilon^{5}\left[\left(3 b \tau-\lambda_{1}\right) \partial_{X} \partial_{T} R\right. \\
& \left.-\frac{V^{\prime \prime \prime}}{12} \partial_{X}^{2} R^{3}\right]=0 .
\end{aligned}
$$

Near the critical point $\left(h_{c}, a_{c}\right),(22)$ is written as

$$
\begin{aligned}
& \varepsilon^{4}\left(\partial_{T} R-g_{1} \partial_{X}^{3} R+g_{2} \partial_{X} R^{3}\right) \\
& \quad+\varepsilon^{5}\left(g_{3} \partial_{X}^{3} R+g_{4} \partial_{X}^{2} R^{3}+g_{5} \partial_{X}^{4} R\right)=0,
\end{aligned}
$$

where

$$
\begin{aligned}
g_{1} & =\left(\frac{V^{\prime}}{6}+\frac{\lambda_{1} b\left(1+b \tau_{c}\right)}{2}+\frac{\left(\lambda_{2}+\lambda_{3}\right)\left(\gamma s^{2}-1\right)}{6}\right. \\
& \left.-\frac{7 b^{3} \tau_{c}^{2}}{6}\right) \\
g_{2} & =-\frac{V^{\prime \prime \prime}}{6}, \\
g_{3} & =\frac{3 b^{2} \tau_{c}}{2}, \\
g_{4} & =\frac{6 b \tau_{c}-2 \lambda_{1}-1}{12} V^{\prime \prime \prime}, \\
g_{5} & =-\frac{23}{8} b^{4} \tau_{c}^{3}+\frac{12 \tau_{c}-4 \lambda_{1}-1}{24} V^{\prime}+\lambda_{1}\left(\frac{5 b^{2} \tau_{c}}{4}\right. \\
& \left.+\frac{5 b^{3} \tau_{c}^{2}}{2}-\frac{\lambda_{1} b\left(1+b \tau_{c}\right)}{2}-\frac{b}{6}\right)+\left(\lambda_{2}+\lambda_{3}\right) \\
& +\frac{\left(12 b \tau_{c}-4 \lambda_{1}\right)\left(\gamma s^{2}-1\right)-\left(\gamma s^{3}-1\right)}{24} .
\end{aligned}
$$

Then a transformation for (23) is shown as follows:

$$
\begin{aligned}
T^{\prime} & =g_{1} T, \\
R & =\sqrt{\frac{g_{1}}{g_{2}}} R^{\prime} .
\end{aligned}
$$

Here, the regularized equation of (23) is obtained:

$$
\begin{aligned}
\partial_{T} R^{\prime} & -\partial_{X}^{3} R^{\prime}+\partial_{X} R^{\prime 3} \\
& +\varepsilon\left(\frac{g_{3}}{g_{1}} \partial_{X}^{2} R^{\prime}+\frac{g_{4}}{g_{2}} \partial_{X}^{2} R^{\prime 3}+\frac{g_{5}}{g_{1}} \partial_{X}^{4} R^{\prime}\right)=0 .
\end{aligned}
$$

Ignoring the $O(\varepsilon)$ term in (26), we get the modified $\mathrm{KdV}$ equation

$$
R_{0}^{\prime}\left(X, T^{\prime}\right)=\sqrt{\mathrm{c}} \tanh \sqrt{\frac{\mathrm{c}}{2}}\left(X-c T^{\prime}\right)
$$

where $c$ is the propagation velocity of the kink wave, which is determined by $O(\varepsilon)$ term. In order to decide the value of $c$, the solvability condition must be satisfied:

$$
\begin{aligned}
& \left(R_{0}^{\prime}, M\left[R_{0}^{\prime}\right]\right) \\
& \quad=\int_{-\infty}^{\infty} d X R^{\prime}{ }_{0}\left(X, T^{\prime}\right) M\left[R_{0}^{\prime}\left(X, T^{\prime}\right)\right]=0,
\end{aligned}
$$

where

$$
M\left[R_{0}^{\prime}\right]=\frac{g_{3}}{g_{1}} \partial_{X}^{2} R^{\prime}+\frac{g_{4}}{g_{2}} \partial_{X}^{2} R^{\prime 3}+\frac{g_{5}}{g_{1}} \partial_{X}^{4} R^{\prime}
$$

After integration, we get the selected speed $c=5 g_{2} g_{3} /$ $\left(2 g_{2} g_{5}-3 g_{1} g_{4}\right)$. Then, the solution of the modified KdV equation is obtained

$$
R(X, T)=\sqrt{\frac{g_{1} c}{g_{2}}} \tanh \sqrt{\frac{c}{2}}\left(X-c g_{1} T\right) .
$$

From (8), we know $V^{\prime}=v_{\max } / 2, V^{\prime \prime \prime}=-v_{\max }$. The amplitude of the kink solution is given by $A=\left[\left(g_{1} c / g_{2}\right)\left(a_{c} / a-1\right)\right]^{1 / 2}$ with

$$
a_{c}=\frac{3\left(v_{\max }+2\left(\lambda_{2}+\lambda_{3}\right)(\gamma-1)\right)^{2}}{2 v_{\max }+4 \lambda_{1}\left(v_{\max }+2\left(\lambda_{2}+\lambda_{3}\right)(\gamma-1)+4\left(\lambda_{2}+\lambda_{3}\right)(\gamma s-1)\right)} .
$$

Through the mKdV equation, the coexisting curves are obtained. The coexisting phase consists of congested phase with high density and freely moving phase with low density. The successive space in freely moving phase is $\Delta x=h_{c}+A$, and that in congested phase is $\Delta x=h_{c}-A$. The coexisting curve and neutral stability curve divide the whole area into stable, metastable, and unstable areas, just as Figure 2 has shown. 


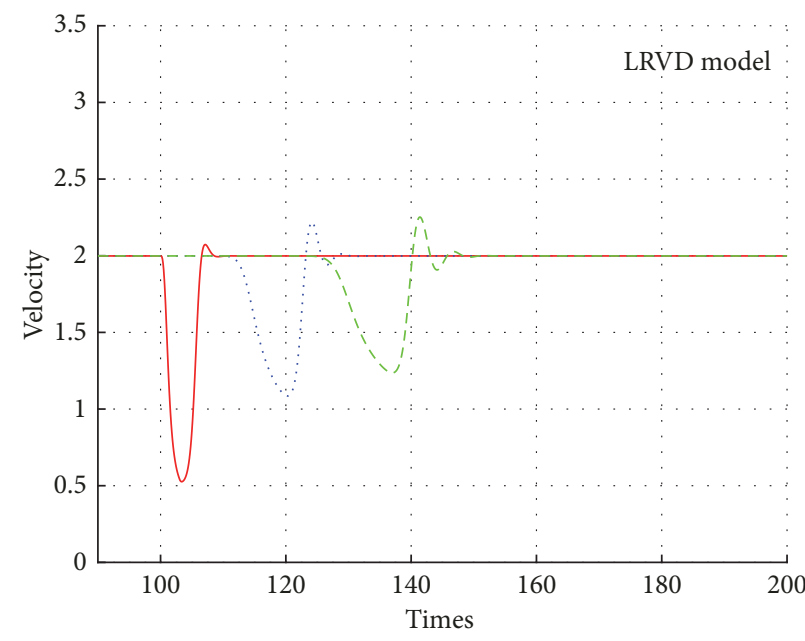

(a)

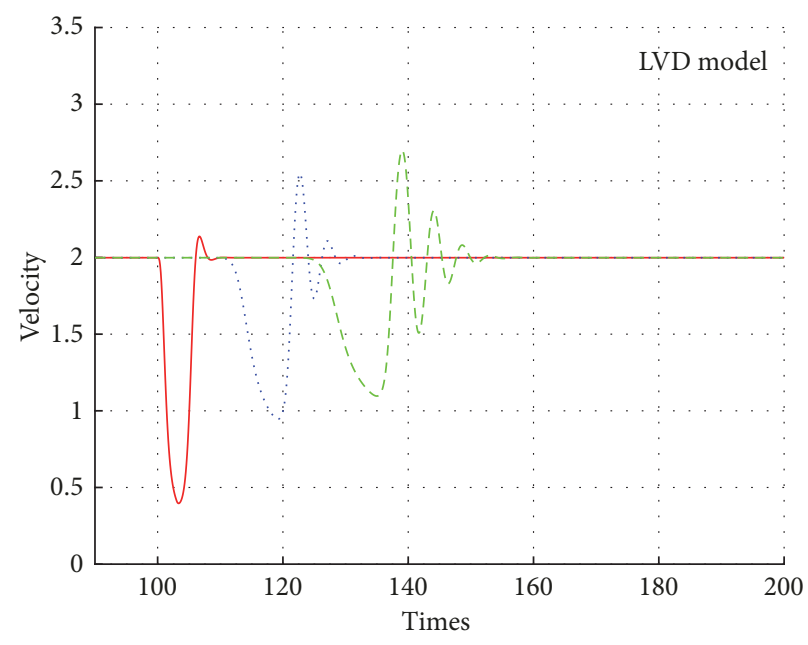

(b)

FIGURE 5: (Color online) speed-time profile of the LRVD model (a) and LVD model (b) after the first car stops from the $100 \mathrm{~s}$ to $103 \mathrm{~s}$. (a) $\lambda_{1}=\lambda_{2}=\lambda_{3}=0.2$ (b) $\lambda_{3}=0, \lambda_{1}=\lambda_{2}=0.2$. As time passes by, the red line represents the speed fluctuation of the $3 \mathrm{rd}$ car behind the first car, the blue line represents the speed fluctuation of the 25 th car, and the green line represents the speed fluctuation of the 50th car.

\section{Simulation}

In this section, we use simulation results to describe the LRVD model. The initial parameters are given as follows:

On the main lane $v_{\max }=4 \mathrm{~m} / \mathrm{s}, h_{c}=4 \mathrm{~m}, a=2 \mathrm{~s}^{-1}$, $v_{n}(0)=2 \mathrm{~m} / \mathrm{s}, N=50$, and $T=0.1 \mathrm{~s}$, where $N$ is the car number and $T$ is the time step.

On the left and right side lanes: $v_{m}^{L}(0)=v_{k}^{R}(0)=$ $2 \mathrm{~m} / \mathrm{s}, N_{L}=N_{R}=50$, and $h_{m}=h_{k}=4 \mathrm{~m}$; all the cars on the two side lanes and the main lane keep the same characteristics during the whole simulation. All the cars are driving at the speed of $2 \mathrm{~m} / \mathrm{s}$ until $100 \mathrm{~s}$ on their own lanes. From $100 \mathrm{~s}$ to $103 \mathrm{~s}$, the leading car on the main lane stops for three seconds as a disruption to the traffic flow, and then the leading car runs later at the recovery speed of $2 \mathrm{~m} / \mathrm{s}$.

Here in the SLVD model, $\lambda_{1}=\lambda_{2}=\lambda_{3}=0.2$. In the LVD model, $\lambda_{1}=\lambda_{2}=0.2, \lambda_{3}=0$. Figure 5 shows the speed fluctuation of the $3 \mathrm{rd}, 25 \mathrm{th}$, and 50th cars after $100 \mathrm{~s}$, when $\lambda_{2}=\lambda_{3}=0.2$ in LRVD model and $\lambda_{2}=0.2, \lambda_{3}=0$ in the LVD model.

When perturbation is set in the stable traffic flow, it will be amplified with time. The traffic jam, go-and-stop wave and speed fluctuation will all appear in the simulation. In Figure 5, the speed fluctuation in LRVD model is smaller than that in LVD model, which means the stability of LRVD model has been improved compared to the LVD model. The cars run with less perturbation effect when considering the relative speed on the other adjacent lane in LRVD model.

To explore the improved stability of LRVD model, we pay attention to the distances between the first car and every following car behind it on the main lane. Figure 6 shows the distance-time patterns of LRVD model and LVD model, from which we realize the leading car' perturbation propagates backward after it stops at 100 s. From Figure 6, we know the
LRVD model recovers its stable state much earlier than LVD model. The distance between the 50th car and the leading car in LVD model recovers the distance in stable state at about $145 \mathrm{~s}$; but the distance between the 50th car and the leading car in LRVD model recovers its stable distances at $141 \mathrm{~s}$. In fact, the traffic flow will recover its stable state with different time, just because of the different stability of the different model. The LVD model needs more time, but LRVD model needs less time. This simulation results mean, under the same traffic situation in real life, the LRVD is more stable than LVD model.

Results of the simulation shown in Figure 6 are described below (reaction time $\tau$ is $0.1 \mathrm{~s}$ ).

(1) When $t=100 \mathrm{~s}$, the first car stops; then all the distances between the first car and each car that follows start decreasing. At that time, the driver of the second car cannot react from the first car's stopping. So all the following cars, from the second car to the last one, go on running at the uniform linear motion on the main lane with the speed of $2 \mathrm{~m} / \mathrm{s}$.

(2) When $t=100.1 \mathrm{~s}$, the second car reacts to the first car stopping and starts to decelerate. Because of the time delay $\tau$, the second driver decides its acceleration value according to the acceleration formula with the relative speed, relative distance, and the second car's speed information and so on at the last time. Here the second driver gets its deceleration value from the relative speed, its own speed, and relative distance with the leading car at $t=100 \mathrm{~s}$. Then, based on the acceleration value, the second driver moves according to the $v_{100.1 \mathrm{~s}}=v_{100 \mathrm{~s}}+a t, x_{100.1 \mathrm{~s}}=x_{100 \mathrm{~s}}+v_{100 \mathrm{~s}} t+(1 / 2) a t^{2}$ from $t=100 \mathrm{~s}$ to $t=100.1 \mathrm{~s}$, by which we get all the relevant parameters value at $t=100.1 \mathrm{~s}$. At $t=100.1 \mathrm{~s}, v_{1}=0, v_{2}<v_{3}$, $v_{3}=2 \mathrm{~m} / \mathrm{s}$, and $\Delta x(2)<\Delta x(3)<4 \mathrm{~m} .(\Delta x(i)=x(i)-x(i-1))$. The remaining cars, from the third car to the last one, go on running at the uniform linear motion with a speed of $2 \mathrm{~m} / \mathrm{s}$. 


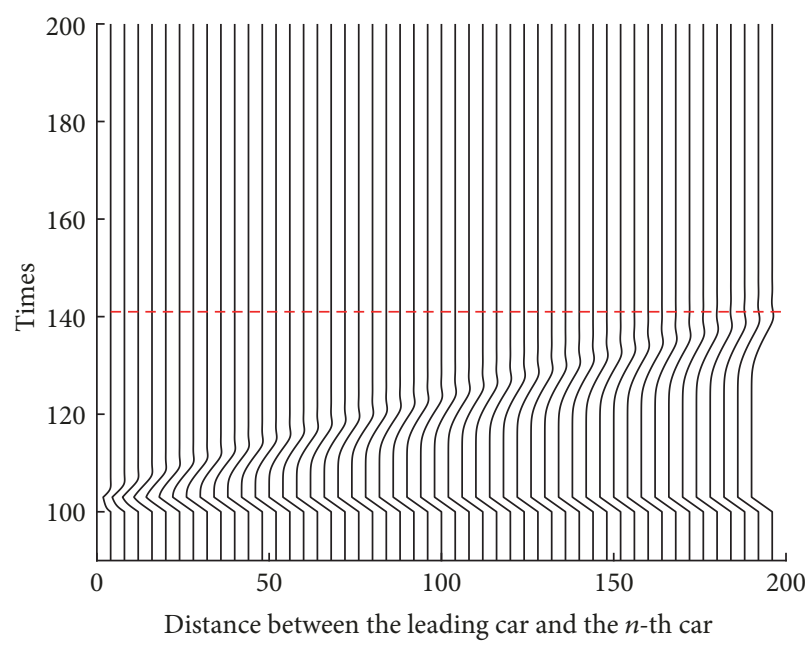

(a)

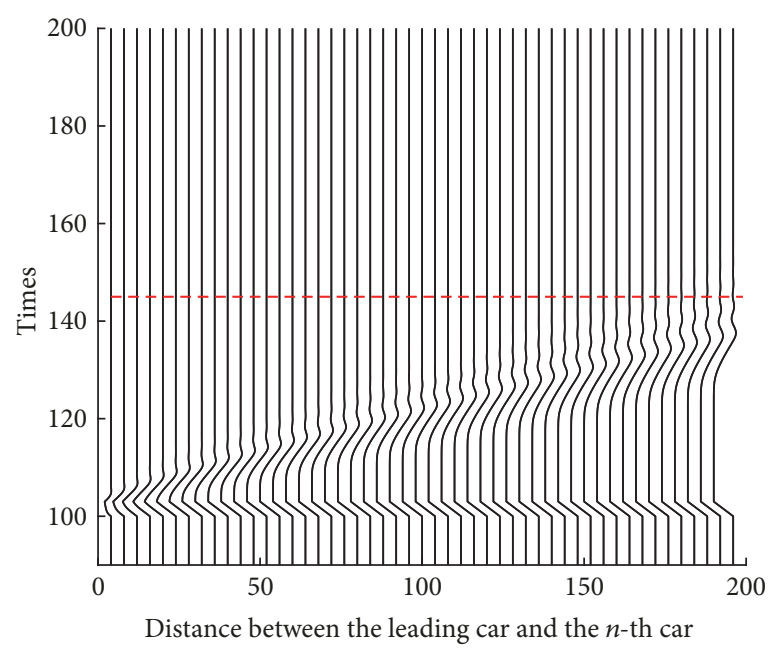

(b)

FIGURE 6: (Color online) distance-time profile of the LRVD model (a) and LVD model (b). The first car stops from $100 \mathrm{~s}$ to $103 \mathrm{~s}$ with (a) LRVD model $\lambda_{1}=\lambda_{2}=\lambda_{3}=0.2$ (b) $\lambda_{3}=0, \lambda_{1}=\lambda_{2}=0.2$. The red line shows the end of the distance change. The LRVD needs $141 \mathrm{~s}$, and LVD model needs $145 \mathrm{~s}$.

(3) When $t=100.2 \mathrm{~s}$, the third car realizes the second car has decelerated, so it starts to decelerate. At this time, $\Delta x(2)<$ $\Delta x(3)<\Delta x(4)<4 \mathrm{~m}, \Delta x(i)=x(i)-x(i-1), a_{1}=0, a_{2}<$ $a_{3}<0$. The remaining cars, from the fourth car to the last one, go on running at the uniform linear motion with a speed of $2 \mathrm{~m} / \mathrm{s}$.

(4) The following $i$ th car decelerates according to the $(i-$ 1)th car ahead deceleration so that $\Delta x(2)<\Delta x(3)<\cdots<$ $\Delta x(i+1)<4 \mathrm{~m}$, and $a_{1}=0, a_{2}<a_{3}<\cdots<a_{i}<0, a_{i+1}=0$, $v_{1}=0, v_{2}<v_{3}<\cdots<v_{i}<2 \mathrm{~m} / \mathrm{s}, v_{i+1}=2 \mathrm{~m} / \mathrm{s}$. During the time interval from $100 \mathrm{~s}$ to $103 \mathrm{~s}$, we may expect 30 cars to be affected by this disturbance. However, the disturbance does not spread so fast: in fact, the cars in front, from the second one to the fifth car, are affected every $\tau$ until $100.4 \mathrm{~s}$ because of $\left|a_{i}\right|>0$. But the speed of the cars, which are behind $j$ th car $(j>i$, here $j=6$ in our simulation), will change much less every $\tau$ time, because $\left|a_{j}\right| \approx 0,\left|a_{j} t\right| \approx 0, v_{j}=v_{j, 0}+a_{j} t$. In this case, the successive distance after $j$ th car even seems to be not changed. But as time passes by, during the $100 \mathrm{~s}$ to $103 \mathrm{~s}$, the cars in front keep decelerating, so the following cars will repeat the same decelerating process above; in fact, the decelerating wave spreads backward.

(5) At $t=103 \mathrm{~s}$, only 11 cars in front decelerate, while all the following cars keep with the same speed of $2 \mathrm{~m} / \mathrm{s}$ and the successive space of $4 \mathrm{~m}$ in the stable state. All the distances between the first car and each following car must decrease during the whole three seconds so that $\Delta x(2)<\Delta x(3)<\cdots<$ $\Delta x(12)<4 \mathrm{~m}$, and $\Delta x(13)=\cdots=\Delta x(50)=4 \mathrm{~m}$. At $t=$ $103 \mathrm{~s}$, we assume that the first car instantaneously recovers its stable speed of $2 \mathrm{~m} / \mathrm{s}$; soon before this instant, all the distances between the first car and each following car have reached their minimum value.

(6) After the first car has recovered the stable speed $(2 \mathrm{~m} / \mathrm{s})$, the second car reacts and begins to accelerate at the $103.1 \mathrm{~s}$, but its speed being lower than $2 \mathrm{~m} / \mathrm{s}, \Delta x(2)$ keeps increasing until $\Delta v(2)=0$. After this time, $\Delta x(2)$ recovers the stable value of $4 \mathrm{~m}$ with some fluctuation in the end.

(7) Later, at $t=103.2 \mathrm{~s}$, the third one realizes the second car begins to increase its speed, but according to the adopted numerical values of the parameters, the simulation shows initially that $v(2)<v(3)$, despite the fact that the third car keeps decelerating while the second car is accelerating until $v(2)=v(3)$. At that time, the 3rd car acceleration is zero, which has reached its minimum value; then, the third car begins to accelerate because of $v(2)>v(3)$, and $\Delta v(3)$ keeps increasing until the third car recovers its stable speed of $2 \mathrm{~m} / \mathrm{s}$. Then, after some fluctuation, $\Delta x(3)$ will recover the stable distance of $4 \mathrm{~m}$, but this phenomenon will happen after $\Delta x(2)$ finishes recovering the same stable distance. From $103 \mathrm{~s}$, the distance between the first car and third car keeps increasing from the minimum value up to reach the stable state with some fluctuation in the end.

(8) The 11 cars in front, which have already decreased their speed, will repeat similar process as shown in part 7 . Take the $i$ th car as an example; the $i$ th car decelerates firstly because of $v(i-1)<v(i)$ until $v(i-1)=v(i)$. At this time, $a_{i}=0$, the successive space $\Delta x(i)$ decreases at the lowest distance. Then, with the $(i-1)$ th car accelerating, $i$ th car keeps accelerating, $v(i-1)>v(i)$. Finally $\Delta x(i)$ keeps increasing up to the stable value of $4 \mathrm{~m}$, which occurs after the $(i-1)$ th car has recovered the same distance with respect to the $(i-2)$ th car.

(9) Other following cars behind the 11th car have not decelerated yet. So, each car has to repeat the same process as 11 cars ahead, in the deceleration phase firstly during $100 \mathrm{~s}$ to $103 \mathrm{~s}$, and after $103 \mathrm{~s}$ keeps decelerating at first till the relative speed increases to zero and begins to accelerate later until reaching the stable state, as parts 7 and 8 have described above. For each following car the same process occurs, so the following car indeed needs more time to react to the behavior of the car ahead, which is in compliance with the kinematic wave theory. 
(10) After $103 \mathrm{~s}$, when each following car begins to decelerate, the distance between the first car and this following car starts to increase at once up to reach the stable state distance with some fluctuation in the end. So the recovery wave in Figure 6 propagates obliquely up and back until the last car recovers the stable state.

\section{Conclusions}

In this paper, we presented a new LRVD model through considering the relative speed in front on the three-lane road. The original OV model has been developed into RV, LVD, and especially LRVD model in this paper, which can portray more complex realistic traffic phenomenon of the cars driving behavior on the middle lane of the threelane road. The linear analysis is studied to get the neutral stability curve. The nonlinear analysis is studied to obtain the mKdV equation and the coexisting curve, by which the area is divided into three regions of stable, metastable, and unstable. The simulation results about distance-time profile are accordant with the analysis. The stability of traffic flow of LRVD model has been proved to be improved compared to the LVD model on two lanes. The LRVD model shows if the drivers on the middle lane pay more attention to more cars on the two adjacent lanes in real life, the middle lane traffic flow is certain to be more stable. All the methods, including the linear, nonlinear analyses, and simulation prove that LRVD model with three lanes indeed stabilize traffic flows much better and capture traffic flow characteristic on the three-lane road in real life more effectively. On the complex three-lane road, if intelligent traffic management system based on the huge traffic data for drivers is applied in the real life, it is very helpful to ensure traffic safety, which is also the trend of transportation development in future.

\section{Conflicts of Interest}

The authors declare that there are no conflicts of interest regarding the publication of this paper.

\section{References}

[1] M. Bando, K. Hasebe, A. Nakayama, A. Shibata, and Y. Sugiyama, "Dynamical model of traffic congestion and numerical simulation," Physical Review E: Statistical, Nonlinear, and Soft Matter Physics, vol. 51, no. 2, pp. 1035-1042, 1995.

[2] M. Bando, K. Hasebe, K. Nakanishi, and A. Nakayama, "Analysis of optimal velocity model with explicit delay," Physical Review E: Statistical, Nonlinear, and Soft Matter Physics, vol. 58, pp. 5429-5435, 1998.

[3] D. Helbing and B. Tilch, "Generalized force model of traffic dynamics," Physical Review E: Statistical, Nonlinear, and Soft Matter Physics, vol. 58, no. 1, pp. 133-138, 1998.

[4] D. Chowhury, L. Santen, and A. Schadschneider, "Statistical physics of vehicular traffic and some related systems," Physics Reports, vol. 329, pp. 199-329, 2000.

[5] R. Jiang, Q. Wu, and Z. Zhu, "Full velocity difference model for a car-following theory," Physical Review E, vol. 64, no. 1, pp. 1-4, 2001.
[6] G. F. Newell, "Nonlinear effects in the dynamics of car following," Operations Research, vol. 9, pp. 209-229, 1961.

[7] Z. Liang, S. Zhong-ke, and L. Tong, "An improved FVD model for intersection," Journal of Transportation Systems Engineering and Information Technology, vol. 13, no. 6, pp. 80-85, 2013.

[8] X. Yu, "Analysis of the stability and density waves for traffic flow," Chinese Physics, vol. 11, no. 11, pp. 1128-1134, 2002.

[9] T. Q. Tang, C. Y. Li, and H. J. Huang, "A new car-following model with the consideration of the driver's forecast effect," Physics Letters A, vol. 374, no. 38, pp. 3951-3956, 2010.

[10] M. Treiber, A. Hennecke, and D. Helbing, "Derivation, properties, and simulation of a gas-kinetic-based, nonlocal traffic model," Physical Review E: Statistical, Nonlinear, and Soft Matter Physics, vol. 59, no. 1, pp. 239-253, 1999.

[11] M. Treiber, A. Hennecke, and D. Helbing, "Congested traffic states in empirical observations and microscopic simulations," Physical Review E: Statistical, Nonlinear, and Soft Matter Physics, vol. 62, no. 2, pp. 1805-1824, 2000.

[12] T. Q. Tang, Y. P. Wang, X. B. Yang, and Y. H. Wu, "A new car-following model accounting for varying road condition," Nonlinear Dynamics, vol. 70, no. 2, pp. 1397-1405, 2012.

[13] T. Q. Tang, W. F. Shi, X. B. Yang, Y. P. Wang, and G. Q. $\mathrm{Lu}$, "A macro traffic flow model accounting for road capacity and reliability analysis," Physica A: Statistical Mechanics and its Applications, vol. 392, no. 24, pp. 6300-6306, 2013.

[14] X.-B. Yang, B.-F. Si, and M. Huan, "Mixed traffic flow modeling near Chinese bus stops and its applications," Journal of Central South University, vol. 19, no. 9, pp. 2697-2704, 2012.

[15] S. Li, Z. Shi, and T. Liu, "An improved model based on FVD model and experimental comparison," Journal of Highway and Transportation Research and Development, vol. 31, no. 2, pp. 109113, 2014.

[16] T. Nagatani, "Stabilization and enhancement of traffic flow by the next-nearest-neighbor interaction," Physical Review E: Statistical, Nonlinear, and Soft Matter Physics, vol. 60, no. 6, pp. 6395-6401, 1999.

[17] G. Yu, P. Wang, X. Wu, and Y. Wang, "Linear and nonlinear stability analysis of a car-following model considering velocity difference of two adjacent lanes," Nonlinear Dynamics, vol. 84, no. 1, pp. 387-397, 2016.

[18] J. Chen, Z. Peng, and Y. Fang, "Effects of car accidents on threelane traffic flow," Mathematical Problems in Engineering, vol. 2014, Article ID 413852, 11 pages, 2014. 


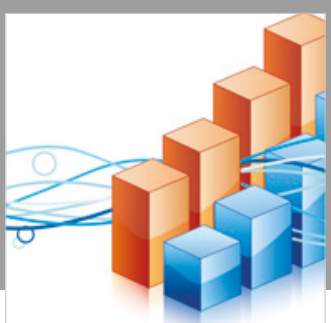

Advances in

Operations Research

\section{-n-m}
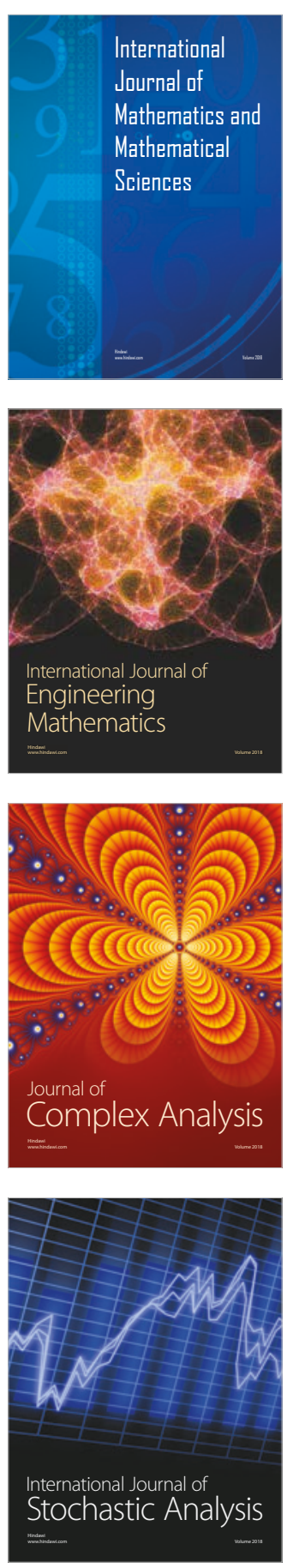
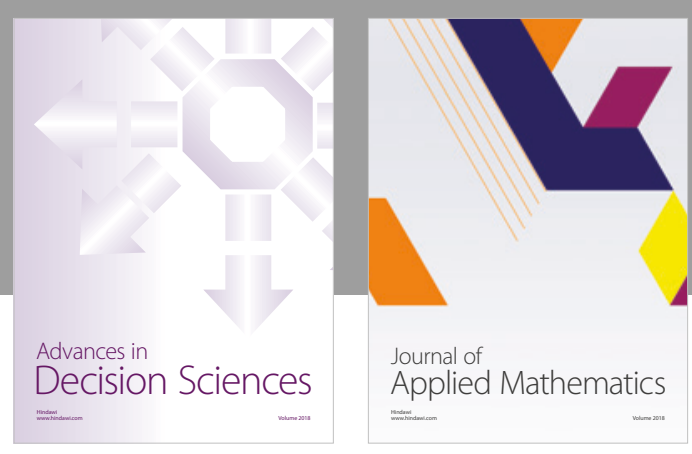

Journal of

Applied Mathematics
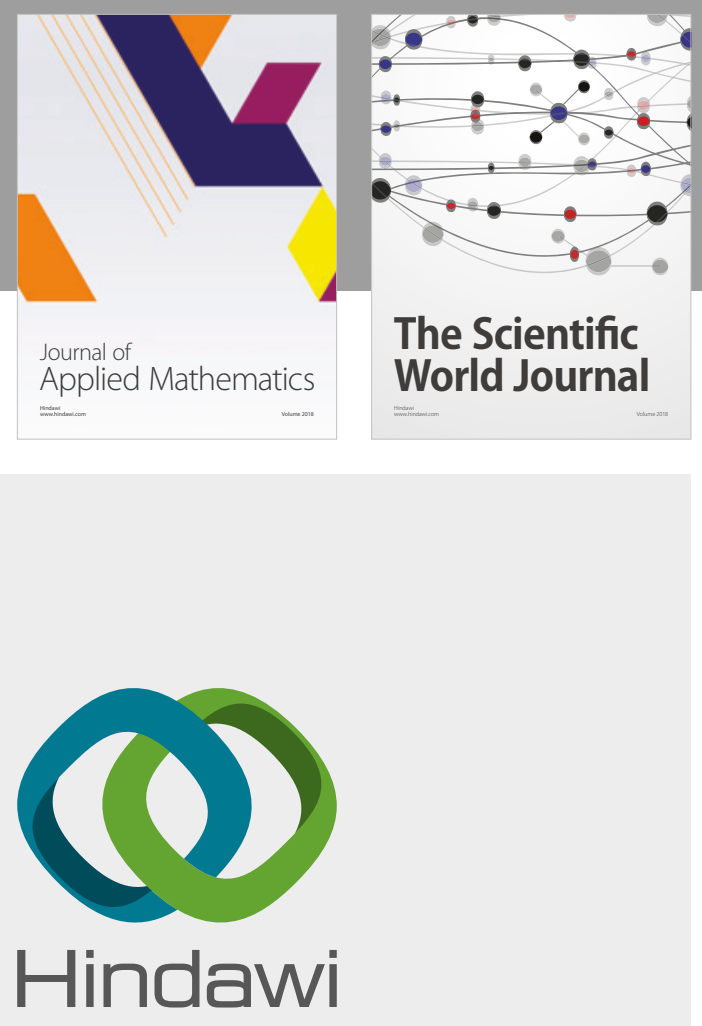

Submit your manuscripts at

www.hindawi.com

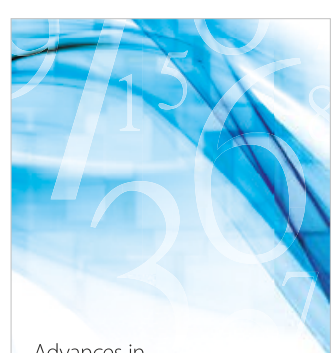

Advances in
Numerical Analysis
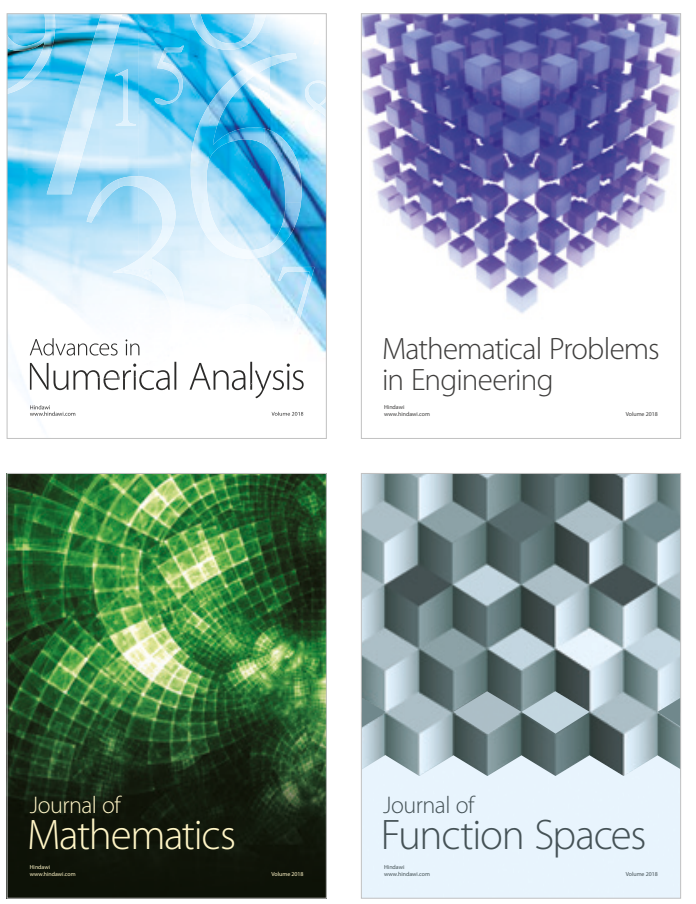

Mathematical Problems in Engineering

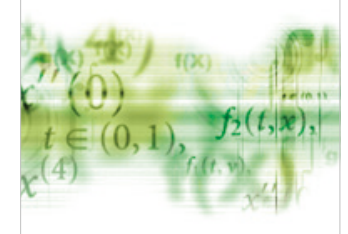

International Journal of

Differential Equations

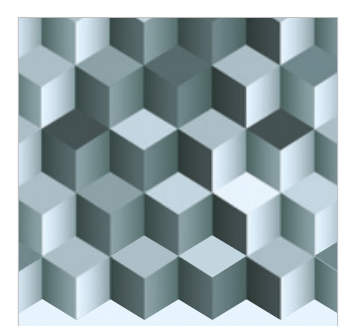

Journal of

Function Spaces
The Scientific

World Journal

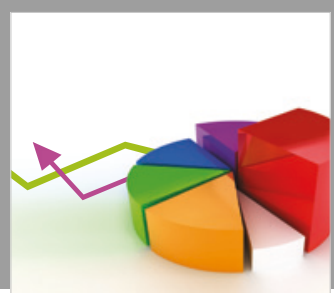

Journal of

Probability and Statistics
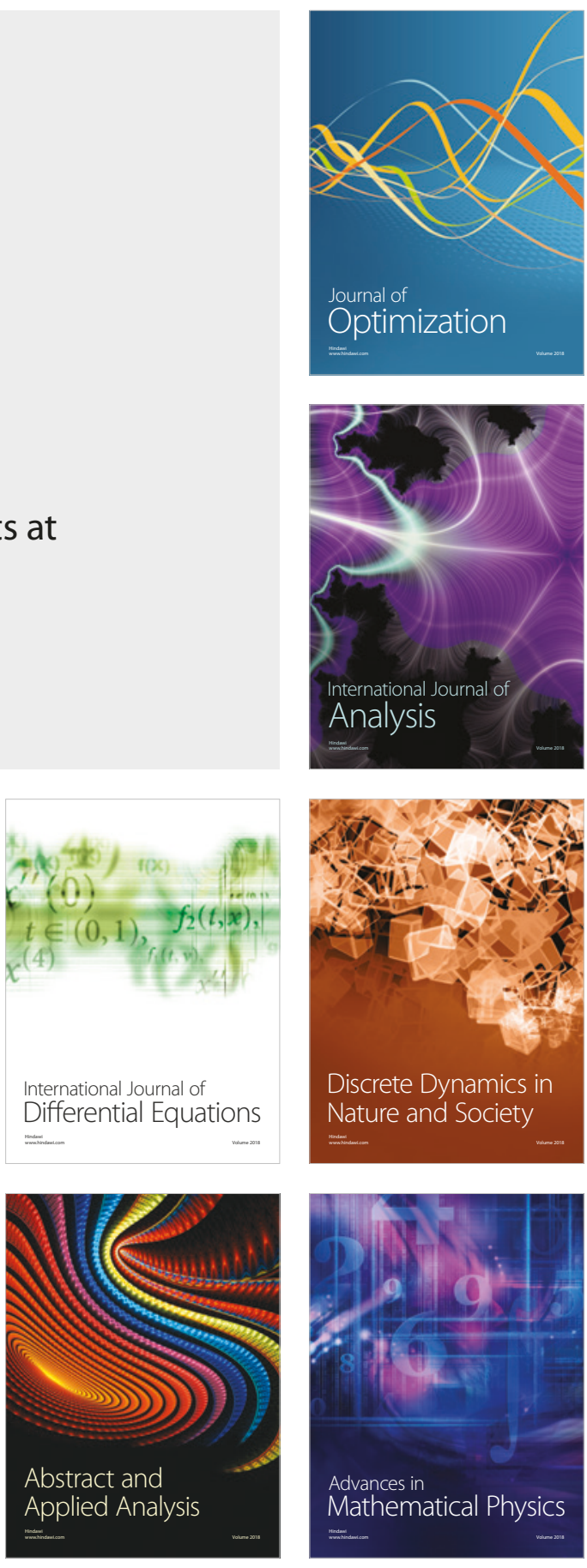